\title{
Sexuality in the 21st Century: Sexual Fluidity
}

\author{
A Ventriglio, D Bhugra
}

\begin{abstract}
Sexuality is a vital component of human life. Sexuality deals with procreation and pleasure. Sexual behaviours and orientations vary and may be related to mental health. Some sexual orientations may be discriminated or censored by religious or political beliefs, and this has a big impact on sexual variations. Frequently, sexual variations are considered to be pathological and need to be medically treated. However, it should be accepted that sexuality includes a continuum of behaviours, thoughts, fantasies, acts, and attractions that are beyond procreation. Modern sexology introduced the concept of gender identity and sexual fluidity to describe how gender and sexual orientation vary and are flexible over time. Healthcare professionals and policy makers should be aware of these new definitions in order to meet health needs of sexually variant people and prevent sexuality discrimination.
\end{abstract}

Key words: Sexual behavior; Sexuality

Antonio Ventriglio, MD, PhD, Department of Clinical and Experimental Medicine, University of Foggia, Foggia, Italy

Dinesh Bhugra, Institute of Psychiatry, King's College, London, UK

Address for correspondence: Dr Antonio Ventriglio, Ospedali Riuniti di Foggia, Servizio Psichiatrico di Diagnosi e Cura, Viale Pinto, 71100, Foggia, Italy.Email: a.ventriglio@libero.it

Submitted: 5 June 2017; Accepted: 10 April 2018

\section{Introduction}

Sexual attraction has historically been seen as rigid even though Kinsey ${ }^{1}$ investigated a gradation of sexual behaviour over 60 years ago. There appears to be confusion between sexual orientation and sexual behaviour. Sexual act is influenced by attraction, availability, fantasy, and the actual act. Historically, sexual orientation has been regarded as division between heterosexual and homosexual, with bisexuality somewhere in the middle. Society defines what sexual behaviours are considered as normal or deviant. There has been a gradual shift towards non-binary classifications of sexual behaviour, sexuality, and gender. Sexuality is broader than sexual acts alone; it is a combination of attraction, thoughts, fantasies, and sexual roles.

Recent debate has raised issues about sexual behaviour in which sexuality is increasingly contextdependent. ${ }^{2,3}$ Humans are sexual beings; human sexuality is a 'vital principle' of each human being and includes feelings and energy of the body with a knowledge and expectations of human intimacy. Sexual behaviours serve functions of pleasure and procreation. Human sexuality in particular carries multiple meanings in different settings. It is important for clinicians to be aware of potential sexual variations in patients. Individual cultures often have strong views on sex and sexuality, and especially on sexual orientation. Individual sexual fantasies may vary from actual sexual acts, and these may differ from actual sexual orientation. For example, a heterosexual male in prison or in a same-sex institution may need heterosexual fantasies to achieve arousal but may perform sexual acts with a male partner.

Recognition of such variations and sexual rights remains controversial. In Italy, there is ongoing political turmoil about these issues involving religious authorities, intellectuals, writers, mass media, and socio-cultural factors. We believe that it is time to classify sexual behaviour in a different way, to take into account increasing sexual fluidity. Diamond $^{4}$ defines sexual fluidity as situation-dependent flexibility in women's sexual responsiveness. For this study, we define sexual fluidity as changes in sexual identity and sexuality as a result of internal and external factors. In the present study, we aim to explore the concept of sexual fluidity and argue that most individuals have a degree of sexually fluid behaviour.

\section{Sexual Orientation}

Sexual orientation has four key components: ${ }^{5,6}$ (1) selfidentified labels (homosexual, bisexual, heterosexual): in the context of sexual fluidity, these labels change and as Tortorella sees himself as label-less. (2) actual sexual behaviour: this depends on availability of individuals and opportunity. (3) self-reported sexual feelings (fantasies and desires): these are personal and may not be acknowledged publicly or even within the sexual act or in a relationship. (4) genital or brain responses (physiologically measured arousal to male or female images): these are probably the most objective observations and assessments. It is important that sexual orientation must be seen in the context of these four components and dimensions.

\section{Human Sexuality and Its variations}

Sexuality forms a core part of an individual identity and 
affects both sexual behaviour and totality of human functioning and is influenced by social attitudes. ${ }^{7}$ Different individuals have varying levels of importance for two aspects of human sexuality (procreative and recreational or pleasurable). These are affected by both cultural norms and cultural expectations. Procreative aspect is a very small part of sexuality, whereas recreational aspects (pleasure and hedonism), intimacy, and sexual activity as socialconnection are more complex components of sexuality.

Bullough $^{8}$ described societies as sex-positive, wherein sex is seen as a pleasure or a pleasurable activity, or as sex-negative, wherein sex is seen as a purely functional procreative activity. Sexuality is experienced and expressed through sexual behaviours and attractions (procreation and erotic aspects) as well as thoughts, fantasies, desires, beliefs, attitudes, values, practices, and roles. ${ }^{9}$ Human sexuality has several components such as biological, physical, psychological, social, and religious. Biological and physical components deal with procreation and reproductive physiology that involve neurological and hormonal responses. ${ }^{10}$ According to Freud, sexuality is at the core of human personality. In this model of childhood development, Freud described psycho-sexual stages every child goes through. This instinctual libido evolving through five phases is primarily based on erogenous zones (oral, anal, phallic, latent, genital). Transiting through these phases is important for an individual's healthy development. Although these remain theories, it has been confirmed that children who experience trauma, stress, or frustration at any stage may develop neurosis or personality disorder later in the life-course. ${ }^{11}$

Historically, sexuality has strongly determined the social life of an individual, because it is related to sexual orientation and sexual behaviour while seeking companionship or pursuing intimacy. Social and cultural contexts of the community significantly influence the integration of each individual based on their own sexuality; sexual identity is thus social. ${ }^{12}$ Meanwhile, sexual identity is personal as it informs an individual sexual existence. As sexual identity is socially constructed, such an interface between the personal and social aspects is the reason for fighting for civil rights related to sexual identity (feminists, gay, lesbian, bisexual, and transgender groups). Such movements have brought significant change in social norms and policies, especially in the West and in high income countries. Among the social and cultural factors, organised religion has played a significant role in 'controlling' sexuality. Religion has continued to modulate attitudes towards sexuality and sexual identity by imposing sexual morality. For example, Catholicism considers sexuality as primarily spiritual and according to the natural law; sexual practice is thus allowed only within the framework of heterosexual marriage, and sexual acts (including masturbation or homosexual acts) are considered sinful as they are not oriented to procreation. ${ }^{13}$

Sexual variation refers to "sexual desires and behaviours outside what is considered to be the normal range, although what is unusual or atypical varies between cultures and from one period to another". ${ }^{14}$ In clinical settings, it is important to differentiate what is atypical and what is seen as deviant or pathological. de Silva ${ }^{14}$ argued that "defining normality is extremely difficult and arbitrary", because this concerns with individual judgments and values even though they occur in the cultural context. In fact, the term 'sexual variations' has replaced sexual deviations or sexual perversions.

Issues related to paraphilias have been discussed elsewhere. ${ }^{15,16}$ Paraphilias carry different perhaps stronger meanings and opprobrium as these were clearly marked and seen as deviant. Sexual variations and behaviours are commonly considered as deviant particularly if they affect others' freedom or cause disease (sexual transmitted diseases, including HIV) or are socially unacceptable (incest, exhibitionism) or prohibited by law (sexual abuse, rape). Social pressures influence policy and dictate what is legal and what falls under the illegal rubric.

Negative attitudes to sexual variations have led to social discrimination: homosexuality was defined as a mental disorder until 1973, before being removed from the DSM following Stonewall riots in New York. It is still considered against the nature's laws in many countries, especially in those where the religious view is strong and affects human rights, ${ }^{6}$ and thus homosexuality and homosexual behaviour is illegal.

\section{Gender and Gender Identity}

Gender is defined as the pattern of characteristics pertaining to masculinity and femininity and has been seen as binary. There is a distinction between biological sex and gender as a role: gender role is the manner in which individuals express their status in the society, ${ }^{17}$ as societal attitudes affect gender, gender roles, and gender role expectations.

Gender identity is the individual experience as well as acknowledgement of one's own gender. All societies are based on a rough gender distinction between male and female and ideals of masculinity and femininity in the framework of a sex/gender model: biological sex - gender identity - gender expression. ${ }^{18}$ Gender is different from biological sex and is a socially constructed identity and thus may be inconsistent with the biological sex attributes. Consequently, an individual is considered outside the sociocultural gender norms, to be defined as gender variant, transgender, or genderqueer or affected by gender identity disorder. ${ }^{19}$

Gender is constructed by acts, society, and psychological factors, and the heterosexual regimen cannot contain the multiplicity of genders. ${ }^{20}$ Increasingly the debate has moved towards creating a non-binary understanding of gender identity. Gender identity and sexuality may well influence each other. The concept of gender spectrum has been proposed to include individuals in the middle space between male and female gender. Some psychologists suggested a model based on gender neutrality, in which 
people are not distinguished by their gender: "perhaps gender neutrality is achieved by combining traits, behaviours, and presentational styles from both genders, and creating a hodge-podge of gender, such that one's sex is unknowable to those on the outside". ${ }^{21}$

\section{Pansexuality}

Pansexuality or omni-sexuality refers to a sexual identity or sexual orientation based on sexual attraction and love towards people of any sex or gender. This means that sex or gender is not relevant in determining the selection of the loved one. ${ }^{22}$ Pansexual people are also defined as genderblind, polysexual, ambisexual. Pansexuality is an extension of the bisexual concept and the gender-binary-system, and is more inclusive. In fact, pansexuals may be attracted to individuals who don't identify themselves as men or women and can be transgender or androgynous. Etymologically, pansexuality could include attraction and love towards "everything" including animals or inanimate objects; it could thus include paraphilias. Sigmund Freud stated that human behaviour is entirely motivated by the sex instinct.

\section{Sexual Fluidity}

The fluidity of gender along with sexual fluidity and flexibility (homo-flexible, hetero-flexible) are newer terms, meaning that sexual attraction may exist and vary within a spectrum or a flux potentially changing over time. ${ }^{23}$

Kinsey $^{1}$ recognised the concept of sexual fluidity when he asked his participants to identify themselves as exclusively heterosexual/exclusively homosexual or largely or predominantly heterosexual thus providing confirmation that sexual behaviours can be situational. He proposed a classification of sexual orientation through a rating scale known as Kinsey scale or Heterosexual-Homosexual Rating Scale. ${ }^{1}$ Kinsey acknowledged a difference between sexual act and sexual orientation. His work has been replicated several times. Although his estimated prevalence of homosexuality was higher than expected largely because of the sample, he set the benchmark for future work.

Gender and sexual orientation may vary and be flexible over time in longitudinal flux. Sexual preferences, attitudes, behaviours, and identity can be flexible to some degree as per the sexual plasticity model..$^{24}$ Sexual fluidity can occur in people who are heterosexual or homosexual and experience a change in their sexual response (hetero-flexible, homoflexible). This is in contrast to the essentialist view that sexual orientation and sexual desire are fundamentally biologically determined and do not change throughout life. In recent studies, people sometimes change their sexual orientation on the base of specific cognitions. ${ }^{24,25}$ Females are more likely than males to believe in sexual fluidity (63\% vs 50\%), even if no significant gender differences in frequency of sexual fluidity are observed.$^{25}$ In fact, many women identify themselves as predominantly heterosexual but find themselves falling deeply in love with one particular woman.

Diamond $^{4}$ argues that as men are able to reproduce most of the time in their lives and their arousability and desire may last longer; therefore, they may pursue sexual contact with desired partners. However, women are reproductive only for a few days of the month during ovulation; they are capable of being aroused by either men or women during the time when their arousability dominates their proceptivity. ${ }^{4}$ This is an elegant and coherent theory of the evolution of female sexual fluidity, although it provides only proximate mechanisms for female sexual fluidity, not an ultimate explanation. ${ }^{26}$ This explanation only answers the 'how' question of proximate mechanisms, not the 'why' question of ultimate functions. ${ }^{26}$

Kuhle and Radtke ${ }^{27}$ hypothesised that the fluid sexual behaviour in women helps them secure resources and care for offspring by promoting the acquisition of allomothering. Similar observation is also made by Kanazawa. ${ }^{26}$ Although some bisexuals may see their bisexuality as a simple transition phase in the fluidity model, as people identified as bisexuals at one stage were especially likely to change sexual orientation and identity throughout the 6-year study. ${ }^{28}$ Sexual fluidity in attractions are the norm rather than the exception among gender minority people (59\%). ${ }^{29}$ However, a larger majority or heteronormative group is needed to further understand the concept and universality of sexual fluidity.

The concept of sexual fluidity could be raised by proponents of conversion therapy (also known as reparative therapy) aimed to change sexual orientation among homosexuals. A clear distinction is needed between sexual fantasy, sexual behaviour, and sexual orientation. Reparative therapy may focus on changing the behaviour but not the innate orientation itself, but it has been reported to be based on faulty assumptions, not effective, and possibly harmful for such individuals. ${ }^{30}$

Kanazawa ${ }^{26}$ proposes that women may have been evolutionarily selected to be sexually fluid in order to allow them to have sex with their cowives in polygynous marriage which may help reduce conflict and provide support as well as increased the likelihood of their reproductive success. He considers sexual fluidity to have three facets: (1) Nonexclusivity: a person is sexually fluid to the extent that she is non-exclusive in her sexual orientation using the four criteria ${ }^{5,6}$ described above. Accordingly, bisexual individuals are sexually more fluid than mostly heterosexuals and homosexuals who in turn are sexually more fluid than strictly heterosexuals and homosexuals. Such a degree of non-exclusivity can be measured by any of the four criteria described earlier. (2) Change: such type of sexual fluidity changes over time or situations using the four criteria as above. ${ }^{5,6}$ Frequency of change may also play a role in recognition of such sexual fluidity. (3) Variance: sexual orientation may well be at variance with that by another definition. A woman is sexually fluid if, for example, she identifies as strictly heterosexual by self-identified label but not necessarily with sexual fantasies and desires about women. The potential problem with the above definition of sexual fluidity is of internal inconsistency. People changing 
from 'mostly heterosexual' to 'strictly heterosexual' back and forth frequently over time will be more sexually fluid than someone who is consistently 'bisexual' over time by the change facet of sexual fluidity, but less sexually fluid by the non-exclusivity facet. ${ }^{26}$

Using the National Longitudinal Study of Adolescent Health, Kanazawa ${ }^{26}$ confirmed the theory's predictions. It was observed that women (but not men) who experience increased levels of sexual fluidity have a larger number of children. This may mean that female sexual fluidity has a major importance in survival of the species. Furthermore, women who married or became mothers earlier on in their lives were more likely to experience higher levels of sexual fluidity later in life. Sexual fluidity is positively correlated with known markers of unrestricted sexual orientation among women, whereas it is negatively correlated with such markers among men. ${ }^{26}$

\section{Sexuality and Human Rights}

Human rights are fundamental and inalienable rights to which an individual is entitled, regardless of sex, origins, ethnicity, religion, language, or any other status. ${ }^{31}$ People who identify themselves in any group of sexual variations should be entitled to equal human rights without any discrimination whatsoever as stated in article 1 of the Universal Declaration of Human Rights: "All human beings are born free and equal in dignity and rights". ${ }^{30}$

Sexual rights have gained substantial acceptance in the human rights community. According to the International Council on Human Rights Policy, sexual rights include the right of all persons to express their sexual orientation, with due regard for the well-being and rights of others, without fear of persecution, denial of liberty, or social interference. ${ }^{32}$ However, only few international legal authorities approve the sexual rights as a category of rights. Policies about rights have addressed only few issues concerning sexual variations and are concentrated in few countries in Europe, United States, Canada, and South Africa. In addition, religion influences the trail for the political approval of civil rights among sexual minorities in some countries. For example, there has been an open debate on the recognition of same-sex civil unions in Italy involving the Roman Catholic Church and politics (legislative proposal by Monica Cirinnà), socio-cultural movements, and mass media. $^{33}$

\section{What Next?}

Apart from clinical medicine and psychiatry, sexuality remains a significant issue in the anthropological, political, sociological, and psychological fields. It is important to understand these concepts in psychiatric settings, as people may be mislabelled and misdiagnosed as having pathologies where none exist. Newer concepts about sexual orientation and variations need to be considered for changing policies and to promote campaigns against stigma and discrimination. For example, in Italy there is ongoing political turmoil around these issues, and intellectuals, writers, mass media, cultural movements are dealing with these new sociological phenomena related to sexuality expression.

If the international legal authorities approve sexual rights as a category of rights, it may facilitate the recognition of sexual categories within local policies at different levels. This would improve the acceptance of sexual minorities and reduce homophobia and transphobia. Psychiatry needs to deal with these new issues to ensure that our patients do not suffer. Various psychiatric societies and organisations, following the American Psychiatric Association in 1973, should clearly state the exclusion of such sexual variations from the diagnostic categories, in order to reduce stigma and pathological consideration of sexual variations. Health care professionals should be trained properly to deal with social discrimination and stigma of lesbian, gay, bisexual, and transgender individuals. Organisations delivering services and support to individuals within the sexual variation spectrum should be encouraged, to improve assessment of emerging health problems associated with the minority condition and stress.

\section{Declaration}

The authors have no conflict of interest to disclose.

\section{References}

1. Kinsey AC, Pomeroy WR, Martin CE. Sexual behavior in the human male. 1948. Am J Public Health 2003;93:894-8. Crossref

2. Wong C. 2 Younger 2 star Tortorella opens up about being sexually fluid. The Huffington Post. 23 June 2016.

3. Kort J. The bisexual dilemma: grief and the loss of gay and lesbian privilege. The Huffington Post. 18 July 2016.

4. Diamond L. Sexual Fluidity: Understanding Women's Love and Desire. Cambridge: Harvard University Press; 2009.

5. Mustanski BS, Chivers ML, Bailey JM. A critical review of recent biological research on human sexual orientation. Annu Rev Sex Res 2002;13:89-140.

6. Wilson G, Rahman Q. Born Gay: The Psychobiology of Sex Orientation. London: Peter Owen; 2005.

7. Kalra G, Ventriglio A, Bhugra D. Sexuality and mental health: issues and what next? Int Rev Psychiatry 2015;27:463-9. Crossref

8. Bullough V. Sexual variance in society and history. Chicago: Chicago University Press; 1976.

9. King BM, Regan P. Human Sexuality Today. 8th ed. Pearson; 2013.

10. Rathus SA, Jeffrey SN, Fichner-Rathus L. Human Sexuality in a World of Diversity. 9th ed. Pearson; 2014.

11. McLeod SA. Psychosexual Stages. Available at: http://www. simplypsychology.org/psychosexual.html. Accessed 31 January 2016.

12. Seidman S, Fischer N, Meeks C. Handbook of the New Sexuality Studies. New York: Routledge International Handbooks; 2006.

13. The Vatican. Catechism of the Catholic Church: Available at: http:// www.vatican.va/archive/ccc_css/archive/catechism/p3s2c2a6.htm. Accessed 31 January 2016.

14. de Silva WP. ABC of sexual health. Sexual variations. BMJ 1999;6;318:654-6. Crossref

15. Bhugra D, Popelyuk D, McMullen I. Paraphilias across cultures: contexts and controversies. J Sex Res 2010;47:242-56. Crossref

16. Ayonrinde O, Bhugra D. Paraphilias and culture. In: Bhugra D, Malhi GS, editors. Troublesome Disguises, 2nd ed. Chichester: Wiley Blackwell; 2015: 199-217.

17. Anke AE. “John Money, PhD”. J Sex Res 2007;44:223-4. Crossref

18. Eller JD. Culture and Diversity in the United States: So Many Ways to Be American. New York: Routledge; 2015:137. Crossref 
19. Blackless M, Besser M, Carr S, et al. Atypical gender development: a review. Int J Transgend 2003;9:29-44.

20. Butler J. Gender Trouble: Feminism and the Subversion of Identity (Subversive Bodily Acts, IV Bodily Inscriptions, Performative Subversions). New York: Routledge; 1999: 179.

21. Reimer D. What does "Gender Neutral" look like?. The Gender Offender. 2012.

22. Jones BE, Hill MJ. Mental Health Issues in Lesbian, Gay, Bisexual, and Transgender Communities. Washington: American Psychiatric Publishing; 2002: 95.

23. Moleiro C, Pinto N. Sexual orientation and gender identity: review of concepts, controversies and their relation to psychopathology classification systems. Front Psychol 2015;6:1511. Crossref

24. Baumeister RF. Gender differences in erotic plasticity: the female sex drive as socially flexible and responsive. Psychol Bull 2000;126:34789. Crossref

25. Katz-Wise SL, Hyde JS. Sexual fluidity and related attitudes and beliefs among young adults with a same-gender orientation. Arch Sex Behav 2015;44:1459-70. Crossref

26. Kanazawa S. Possible evolutionary origins of human female sexual fluidity. Biol Revs Camb Philos Soc 2017;92:1251-74. Crossref
27. Kuhle BX, Radtke S. Born both ways: the alloparenting hypothesis for sexual fluidity in women. Evol Psychol 2013;11:304-23. Crossref

28. Savin-Williams RC, Joyner K, Rieger G. Prevalence and stability of self-reported sexual orientation identity during young adulthood. Arch Sex Behav 2012;41:103-10. Crossref

29. Katz-Wise SL, Reisner SL, Hughto JW, Keo-Meier CL. Differences in sexual orientation diversity and sexual fluidity in attractions among gender minority adults in Massachusetts. J Sex Res 2016;53:7484. Crossref

30. Haldeman DC. The pseudo-science of sexual orientation conversion therapy. Available at: http://drdoughaldeman.com/doc/PseudoScience.pdf. Accessed 31 January 2016.

31. The United Nations. What are human rights? Available at: http://www. ohchr.org/en/issues/pages/whatarehumanrights.aspx. Accessed 31 January 2016.

32. International Council on Human Rights Policy. Available at: http:// www.ichrp.org/files/reports/47/137_web.pdf. Accessed 31 January 2016.

33. Italian Senate. Atto Senato n.14, XVII Legislatura. Available at: https://www.senato.it/leg/17/BGT/Schede/Ddliter/testi/39314_testi. htm. Accessed 31 January 2016. 